\title{
Simulation-Based Training of the Rapid Evaluation and Management of Acute Stroke (STREAM) - A Prospective Single-Arm Multicenter Trial
}

OPEN ACCESS

Edited by:

Bryan G. Young,

London Health Sciences

Centre, Canada

Reviewed by:

Minjee Kim

Northwestern University, United States Alessandra Splendiani,

University of L'Aquila, Italy

*Correspondence:

Waltraud Pfeilschifter

waltraud.pfeilschifter@kgu.de

${ }^{+}$See list of Collaborators at the end of the article

Specialty section:

This article was submitted to Neurocritical and Neurohospitalist

Care,

a section of the journal

Frontiers in Neurology

Received: 20 February 2019

Accepted: 23 August 2019

Published: 11 September 2019

Citation:

Bohmann FO, Kurka N, du Mesnil de Rochemont R, Gruber K, Guenther J,

Rostek P, Rai H, Zickler P, Ertl M, Berlis A, Poli S, Mengel A, Ringleb P,

Nagel S, Pfaff J, Wollenweber FA,

Kellert L, Herzberg M, Koehler $L$, Haeusler KG, Alegiani A, Schubert C, Brekenfeld C, Doppler CEJ, Onur OA, Kabbasch C, Manser T, Pfeilschifter W and STREAM Trial Investigators (2019)

Simulation-Based Training of the Rapid Evaluation and Management of

Acute Stroke (STREAM) - A

Prospective Single-Arm Multicenter

Trial. Front. Neurol. 10:969.

doi: 10.3389/fneur.2019.00969
Ferdinand O. Bohmann ${ }^{1}$ Natalia Kurka ${ }^{1}$, Richard du Mesnil de Rochemont ${ }^{2}$, Katharina Gruber ${ }^{1}$, Joachim Guenther ${ }^{3}$, Peter Rostek ${ }^{4}$, Heike Rai ${ }^{1}$, Philipp Zickler ${ }^{5}$, Michael Ert/ ${ }^{5}$, Ansgar Berlis ${ }^{6}$, Sven Poli ${ }^{7,8}$, Annerose Mengel ${ }^{7,8}$, Peter Ringleb ${ }^{9}$, Simon Nagel $^{9}$, Johannes Pfaff ${ }^{10}$, Frank A. Wollenweber ${ }^{11}$, Lars Kellert ${ }^{11}$, Moriz Herzberg ${ }^{12}$, Luzie Koehler $^{13}$, Karl Georg Haeusler ${ }^{14,15}$, Anna Alegiani ${ }^{16}$, Charlotte Schubert ${ }^{16}$, Caspar Brekenfeld ${ }^{17}$, Christopher E. J. Doppler ${ }^{18}$, Oezguer A. Onur ${ }^{18}$, Christoph Kabbasch ${ }^{19}$, Tanja Manser ${ }^{20}$, Waltraud Pfeilschifter ${ }^{1 *}$ and STREAM Trial Investigators ${ }^{\dagger}$

${ }^{1}$ Department of Neurology, University Hospital Frankfurt, Frankfurt, Germany, ${ }^{2}$ Institute for Diagnostic and Interventional Neuroradiology, University Hospital Frankfurt, Frankfurt, Germany, ${ }^{3}$ Department of Neurology, Klinikum Hanau, Hanau, Germany, ${ }^{4}$ NICU Nursing Staff, University Hospital Frankfurt, Frankfurt, Germany, ${ }^{5}$ Department of Neurology and Clinical Neurophysiology, Universitätsklinikum Augsburg, Augsburg, Germany, ${ }^{6}$ Department for Diagnostic and Interventional Radiology and Neuroradiology, Universitätsklinikum Augsburg, Augsburg, Germany, ${ }^{7}$ Department of Neurology With Focus on Neurovascular Diseases and Neurooncology, University Hospital Tübingen, Tübingen, Germany, ${ }^{8}$ Hertie Institute for Clinical Brain Research, University of Tübingen, Tübingen, Germany, ${ }^{9}$ Department of Neurology, University Hospital Heidelberg, Heidelberg, Germany, ${ }^{10}$ Department of Neuroradiology, University Hospital Heidelberg, Heidelberg, Germany, ${ }^{11}$ Department of Neurology, Ludwig Maximilians-University, Munich, Germany, ${ }^{12}$ Department for Diagnostic and Interventional Neuroradiology, Ludwig Maximilians-University, Munich, Germany, ${ }^{13}$ Department of Neurology, University Hospital Leipzig, Leipzig, Germany, ${ }^{14}$ Centre for Stroke Research Berlin, Charité-Universitätsmedizin Berlin, Berlin, Germany, ${ }^{15}$ Department of Neurology, Universitätsklinikum Würzburg, Würzburg, Germany, ${ }^{16}$ Department of Neurology, University Medical Centre Hamburg Eppendorf, Hamburg, Germany, ${ }^{17}$ Department of Diagnostic and Interventional Neuroradiology, University Medical Centre Hamburg Eppendorf, Hamburg, Germany, ${ }^{18}$ Department of Neurology, University Hospital Cologne, Cologne, Germany, ${ }^{19}$ Department of Neuroradiology, University Hospital Cologne, Cologne, Germany, ${ }^{20}$ School of Applied Psychology, FHNW University of Applied Sciences and Arts Northwestern Switzerland, Olten, Switzerland

Introduction: Acute stroke care delivered by interdisciplinary teams is time-sensitive. Simulation-based team training is a promising tool to improve team performance in medical operations. It has the potential to improve process times, team communication, patient safety, and staff satisfaction. We aim to assess whether a multi-level approach consisting of a stringent workflow revision based on peer-to-peer review and 2-3 one-day in situ simulation trainings can improve acute stroke care processing times in high volume neurocenters within a 6 months period.

Methods and Analysis: The trial is being carried out in a pre-test-post-test design at 7 tertiary care university hospital neurocenters in Germany. The intervention is directed at the interdisciplinary multiprofessional stroke teams. Before and after the intervention, process times of all direct-to-center stroke patients receiving IV thrombolysis (IVT) and/or endovascular therapy (EVT) will be recorded. The primary outcome measure will be the "door-to-needle" time of all consecutive stroke patients directly admitted to 
the neurocenters who receive IVT. Secondary outcome measures will be intervention-related process times of the fraction of patients undergoing EVT and effects on team communication, perceived patient safety, and staff satisfaction via a staff questionnaire.

Interventions: We are applying a multi-level intervention in cooperation with three "STREAM multipliers" from each center. First step is a central meeting of the multipliers at the sponsor's institution with the purposes of algorithm review in a peer-to-peer process that is recorded in a protocol and an introduction to the principles of simulation training and debriefing as well as crew resource management and team communication. Thereafter, the multipliers cooperate with the stroke team trainers from the sponsor's institution to plan and execute 2-3 one-day simulation courses in situ in the emergency department and $\mathrm{CT}$ room of the trial centers whereupon they receive teaching materials to perpetuate the trainings.

Clinical Trial Registration: STREAM is a registered trial at https://clinicaltrials.gov/ct2/ show/NCT03228251.

Keywords: CRM, thrombolysis (tPA), stroke, emergency care, simulation training

\section{INTRODUCTION}

The benefits of acute stroke therapy critically depend on the time to recanalization of the occluded brain vessel. This has been consistently shown for intravenous thrombolysis (IVT) (1) and endovascular thrombectomy (EVT) (2), nowadays applied to 525 or $5-10 \%$ of all patients suffering from an ischemic stroke, respectively. Each minute lost reduces therapeutic efficacy and increases the risk of complications $(3,4)$. When implementing these therapies into daily clinical practice, stroke teams have to strive for optimal process times in order to translate the benefits shown in clinical trials into good outcomes for the patients. Since the widespread introduction of EVT, the chain of acute stroke treatment has become more complex. The patient is cared for by an interdisciplinary team (5) and has to undergo several handovers with possible interface problems.

As treatment delays, errors or misjudgements may put the patient at risk, the initial assessment, diagnostic procedures and therapeutic decisions have to be as fast as possible without losing accuracy. The decision to start IVT and/or EVT should be completed within $<30 \mathrm{~min}$, which requires a well-functioning workflow including initial assessment, blood sampling, the organization of further processes, transport, and radiological diagnostics. Recent reports show that excellent process times can be reached at high volume centers but not as reliably at the majority of smaller stroke units worldwide $(6,7)$. So far, reports on workflow improvement in the setting of acute stroke care have focussed on streamlining of the process but how the teams that work along these processes are actually trained has not been detailed $(8,9)$. It is a well-recognized phenomenon that time pressure increases the risk of medical errors and puts strain on team communication. Complications and adverse events during medical treatment are a frequent problem (10).
In 2010, up to every fourth patient treated in a hospital may have suffered from avoidable medical complications $(11,12)$. Medical complications caused by human failure are estimated to represent $3-4 \%$ of the health expenditure. Approximately $70 \%$ of these cases are attributed to human factors, such as miscommunication, forgotten or falsely executed orders, mixups, or wrong decisions (13).

One means to counteract the deleterious effect of time pressure on team communication is crew or crisis resource management (CRM), which is a work and training philosophy combining different strategies for teams focusing on nontechnical skills in high-reliability environments where human error can have a devastating effect (14). CRM concepts, which were originally designed for aviation, have been modified and adapted to a wide range of high-reliability environments where people have to make time-critical wide-ranging decisions, such as in emergency medicine. Previously, the trial sponsor (University Hospital Frankfurt, Germany) designed a stroke team training for the interdisciplinary multiprofessional team of its neurocenter using an in situ simulation along the entire chain of acute stroke care from the paramedics' handover to the beginning of the neurovascular intervention. We introduced the principles of CRM into our stroke care protocol with a focus on communication skills, a defined team, a binding algorithm involving the specific division of tasks and predefined "check points" for a brief team time-out (15). In order to acquaint the staff with the algorithm, we implemented monthly simulation training with a high-fidelity manikin. This intervention was highly efficient (16). We transferred a trimmed-down version of the intervention-relying on a peer-to-peer review of the respective acute stroke algorithms and a one-time simulation training-to our regional stroke network consisting of stroke units of different certification levels where it significantly reduced 
the network-wide median door-to-needle time in a pre-test-posttest analysis (17).

The STREAM trial is directed at high level stroke centers with 24/7 EVT capacity in a controlled prospective pre-test-posttest design. It will assess the immediate effect of a composite intervention that includes a peer-to-peer review of each center's acute stroke algorithm, which defines the team's composition and details the specific tasks of each team member. This is in combination with a short course of repetitive stroke team simulation training. We hypothesize that the implementation of a stroke team algorithm (defined team with precisely defined tasks), applying the principles of crew resource management $(\mathrm{CRM})$ and stroke team simulation training with a focus on efficient teamwork and communication will improve the processing time, patient safety, and staff satisfaction.

\section{METHODS AND ANALYSIS}

\section{Design}

STREAM is a prospective single-arm multicenter trial with a pre-test-post-test design (Figure 1) and central data monitoring, employing individual patients' case report forms (Supplemental Data 1) to record the baseline variables, processing time, time-consuming items in the acute stroke workflow, and the team's composition. Additionally, staff questionnaires will be used to capture satisfaction regarding interdisciplinary teamwork, safety climate, stress recognition and error handling as well as general job satisfaction and identification within the workplace.

\section{Interventional Methods}

During a 3-months pre-test period, the participating seven stroke centers (tertiary care university hospitals with 24/7 thrombectomy, well-comparable to other tertiary stroke care centers) will record the data of all consecutive stroke patients directly admitted to the emergency departments. The participating centers are University Hospital Augsburg, University Hospital Tübingen, University Hospital Heidelberg, Ludwig Maximilians-University Munich, Center for Stroke Research Berlin Charité, University Medical Center Hamburg Eppendorf, University Hospital Cologne. University Hospital Frankfurt (Goethe University) is the sponsor of the trial and is not participating in the trial. All consecutive patients undergoing acute recanalization therapy (IVT and/or EVT) will be enrolled. Pre-test staff satisfaction and patient safety will be measured using a 67-item questionnaire, based on the Safety Attitudes Questionnaire (SAQ), a validated instrument used to measure the patient safety climate in clinical areas $(18,19)$. After the pre-test period (observation phase 1), the data acquisition will be paused for 6 months during which the intervention will take place.

We will perform a multi-level intervention in close cooperation with three "STREAM multipliers" per center. We recommended the centers to nominate one stroke neurologist, one neurointerventionalist and one nurse of the emergency department but left the final decision about the composition of the multiplier team to each center. The first step of the intervention will be a central meeting at the sponsor's institution (to accommodate shift plans etc., we will conduct two meetings with identical programmes and final written distribution of all protocols). The purpose of this first meeting will be a critical revision of each center's acute stroke algorithm in a peer-to-peer process that is captured in a protocol and provided to each center as well as a familiarization of the multiplier team with stroke simulation. To this end, there will be a demonstration of stroke team simulation and a seminar on basic principles of simulation training and debriefing as well as CRM and team communication. The protocol on each center's algorithm will encourage the implementation of an interdisciplinary stroke team with a binding and precise definition and assignment of tasks. Thereafter, the multipliers will cooperate with the sponsor's stroke team trainers to organize 2-3 simulationbased team trainings with a high-fidelity manikin in situ in each center's emergency department and CT room. Two of the training sessions shall be conducted by the stroke team trainers from the sponsor while the third training session will be encouraged to be led by one of the multipliers. This is with the aim of establishing the concept of stroke team training and simulation on a regular basis. The centers will be provided teaching materials for use at their institution and their regional stroke networks. The intervention shall be completed within 6 months.

During the following 3-month post-test period (observation phase 2), the participating seven centers will record the process times of all consecutive direct-to-center patients receiving recanalization therapy (IVT and/or EVT). Post-interventional staff satisfaction and perceived patient safety will be measured with a 67 -item questionnaire.

\section{Selection of Centers and Subjects}

Centers were selected after a site selection screening taking into account the number of recanalization therapies per year, median process times and the readiness to participate with dedication in the intervention. Randomization into an instant and a delayed intervention cohort would be desirable. However, due to the limited number of stroke centers $(n=7)$ and their heterogeneity, this approach is not feasible. Therefore, all seven centers will undergo the intervention and observation phases simultaneously and a comparison will be made between the pre-test-post-test results of all seven centers grouped together.

All patients over the age of 18 years who are admitted directly to the participating centers and receive IVT (primary endpoint: door-to-needle time) in the context of an acute stroke are eligible irrespective of whether they receive additional EVT or not. If EVT is performed, then the EVT-specific processing time will also be acquired (secondary endpoints). Written informed consent will be obtained from all patients or their legal representatives before the transfer of data to the sponsor. Patients who had been secondarily transferred to the stroke centers for EVT ("drip-and-ship") and patients suffering in-hospital stroke will be excluded from the trial.

Observation phase 1: All direct-to-center stroke patients receiving thrombolysis and/or thrombectomy in the 3 months before the intervention phase. 


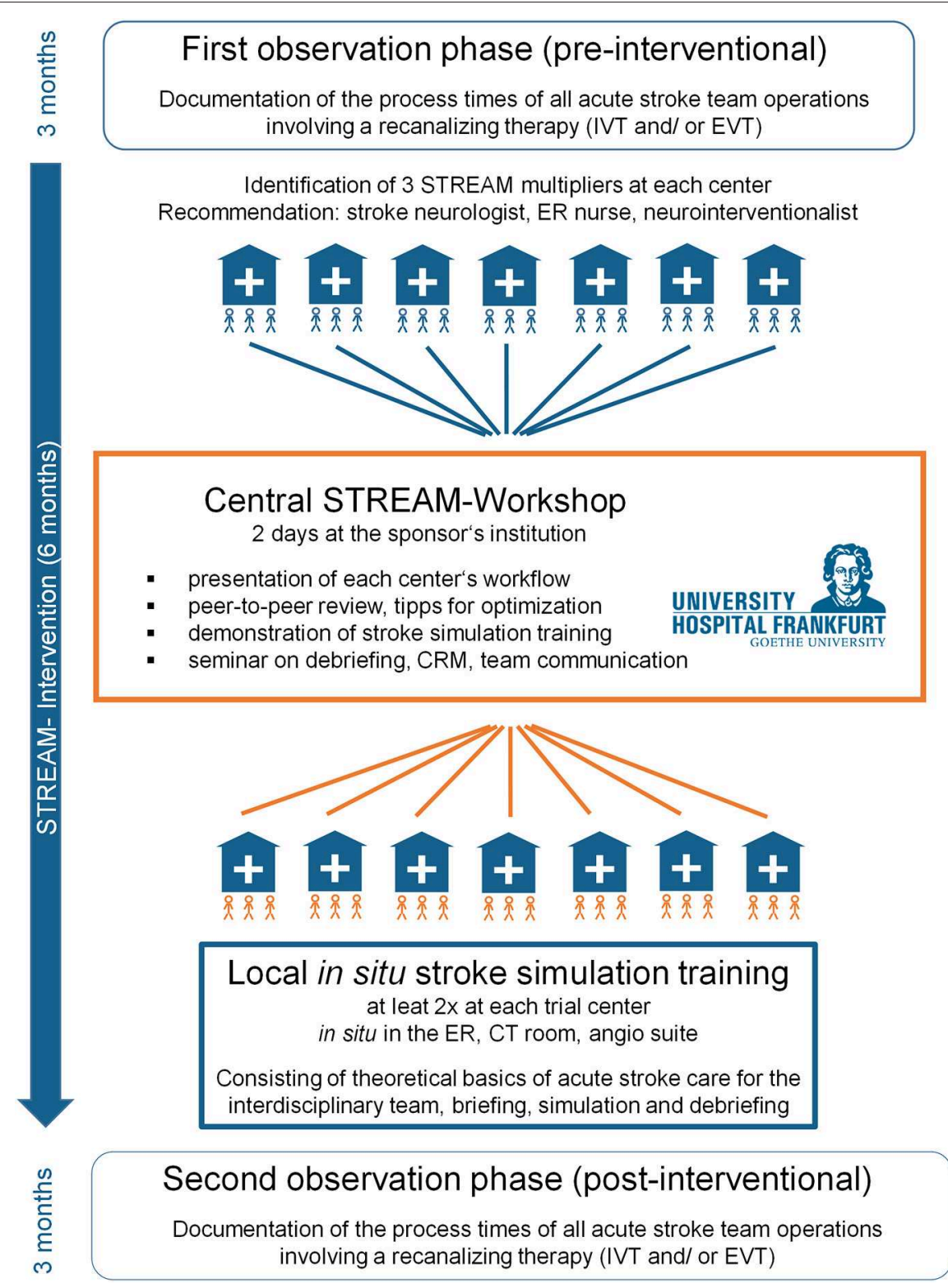

FIGURE 1 | Timeline of the STREAM trial.

Observation phase 2: All direct-to-center stroke patients receiving thrombolysis and/or thrombectomy in the 3 months after the intervention phase.

\section{Data Analysis}

The predefined primary endpoint is the median time from the arrival of the stroke patients until the administration of the alteplase bolus ("door-to-needle" time) of all consecutive directto-center stroke patients in observation periods 1 and 2 is the primary endpoint.

The predefined secondary endpoints are median EVT process time ("door-to-angio" comprising the critical handover to the anesthesia or neurocritical care team and "door-to-groin" comprising the entire process up to the endovascular procedure). Besides that, secondary haemorrhagic transformation (symptomatic or asymptomatic) will be recorded as a safety endpoint. The team composition, as well as any time-consuming processes, will also be recorded as crucial variables targeted by our intervention. Via the questionnaires, we aim to assess interdisciplinary teamwork, the safety climate, stress recognition and error handling as well as staff satisfaction and identification with the workplace during both observation periods.

All data specified in the trial protocol will be documented in standardized Case Report Forms (CRFs, Supplemental Data 1) by the participating stroke centers. Data validation, including the control of completeness, consistency and plausibility of the data 
documented in the CRF, is the responsibility of the local principal investigator (PI) and the documenting investigators. HR, who is a clinical trials coordinator at the sponsor's institution, will carry out source data verification of $15 \%$ of recruited patients.

A pilot trial on the efficacy of a fixed stroke team algorithm and regular simulation-based stroke team training in our regional stroke network consisting of 7 stroke units showed a shortening of the network-wide median "door-to-needle" time by $12 \mathrm{~min}$ (43, IQR 30-60 to 31 , IQR 24-42 $\mathrm{min}$ ). A priori sample size calculation based on this data yielded a minimal number of 110 patients in each group to prove the comparative efficacy of the composite intervention on the primary endpoint with a statistical power of 0.8 and a type 1 error probability of $<0.05$. The seven tertiary care stroke centers perform $80-250$ thrombolyses per year and should jointly be able to recruit 200 patients in each intervention phase considering the potential dropout rate for missing reports or informed consent in $1 / 3$ of the patients. The centers will be explicitly motivated to record all consecutive patients and they will be obliged to prove that adequate efforts have been made via a monitored screening log.

We will present the median and 25-75 percent interquartile ranges (IQR) of the process times and test differences between the intervention phases for statistical significance via the Wilcoxon-Mann-Whitney Test. The answers provided within the questionnaire capturing satisfaction with the interdisciplinary teamwork, safety climate, stress recognition and error handling, as well as staff satisfaction and identification with the workplace, will be recorded using an ordinal 5-point Likert scale.

\section{DISCUSSION}

The STREAM trial will evaluate the effect of a five-step intervention for acute stroke therapy on process times, staff satisfaction and perceived patient safety. The intervention aims at optimizing and standardizing stroke treatment by applying aspects of crew resource management (CRM) and simulation training adapted to acute stroke care. It may provide evidence of efficacy for this composite intervention that could pave the way for the implementation of structured team trainings for acute stroke care, as they are already practiced in other medical disciplines.

In high-reliability environments where decisions are timecritical, structured algorithms based on the principles of crewresource management help to improve patient safety. Once developed, these SOPs have to be introduced to the entire staff. We hypothesize that simulation training will facilitate the transfer of the new algorithms by allowing experiential learning and self-reflection. To evaluate the safety of our intervention, which aims at accelarating IVT, we will capture intra- and extracranial haemorrhagic complications and will compare the frequency between the pre- and post-interventional phases.

Structured team training based on crew resource management (CRM) has been successfully implemented into emergency medicine, intensive care and surgery. In these contexts, training has led to a significant fall in the mortality rate (up to $18 \%)(20$, 21). To our knowledge, there have been only a few reports on the effect of simulation training on process times in acute stroke care. Existing studies rely on one-time interventions at a simulation facility (22), trained hospitals with little prior experience (23) and/or used retrospective case analyses as a comparator $(23,24)$. We have previously applied the stroke team approach to the sponsor's stroke admissions team (16) and consequently to our regional stroke network (17), which at the time consisted of seven stroke units. In that study, we found that the intervention (algorithm design, introducing CRM, and simulation training) was effective in reducing the network wide door-to-needle time from a median of $43 \mathrm{~min}$ (IQR $30-60 \mathrm{~min}$ ) to $31 \mathrm{~min}$ (IQR 24-42 $\mathrm{min}$ ) (16) and door-to-groin-time from $59 \mathrm{~min}$ (IQR 35$102 \mathrm{~min}$ ) to $43 \mathrm{~min}$ (IQR 28-81 $\mathrm{min}$ ) (25). However, these studies were limited to a regional network consisting of heterogeneous stroke units of different sizes with and without the capacity for EVT. The multicentric study in the stroke network relied on data entry by the local investigators without central source data verification. By testing the stroke team concept in seven high volume academic tertiary care stroke centers with the 24/7 capacity for thrombectomy in a multicentric pre-test-post-test study, we aim to control some of these limitations and measure the effectiveness of the intervention more reliably. A cluster randomization by center would have been desirable, but the heterogeneity in between centers (e.g., hospital infrastructure, rural vs. urban catchment area, case load, and percentage of dripand-ship patients) does not permit this. Therefore, we opted for a prospective pre-test-post-test design with central source data monitoring.

Another question is the scalability of our composite intervention. In our regional stroke network, stroke team training could be introduced on a regular basis following the above-named effectiveness study (16). So far, trainings are carried out as part of the cooperation between stroke team trainers from the university hospital with the senior staff of local stroke units. Obstacles to a decentralized implementation of an independent regular training at each site include time and access to a qualification programme for stroke team trainers and to stroke specific teaching materials such as simulation equipment, slidekits and scripted scenarios. If the STREAM trial demonstrates effectiveness of the intervention, it may further promote stroke team simulation as a versatile tool for the education of interprofessional teams. Since all hospitals participating in this trial are part of and often coordinate regional stroke networks, the intervention could be secondarily propagated to these stroke networks. From the feedback of the participating centers, we hope to get an insight into the possible difficulties faced in the implementation processes.

We acknowledge that there are a few limitations that we could not circumvent when designing this trial: randomization is not possible due to the limited number of centers. Since we targeted high volume centers with a median door-to-needle time of $30 \mathrm{~min}$ and below, it will be challenging to show an improvement in the primary endpoint. The success of the intervention will crucially depend on the cooperation of all involved medical disciplines and professionals-a factor that is not easy to foresee in the planning phase of a trial. It will probably not be possible to train all staff members involved in acute stroke therapy during 
the three in situ simulations at each center, so we will have to rely on a snowball effect. Notably, including stroke patients who are able to provide written informed consent or stroke patients with a legal representative implies a selection bias. Finally, it will not be possible to discern the impact of the different levels of our intervention.

Nevertheless, we think that the results of this pragmatic and rigorously controlled trial of workflow-improvement and in situ simulation could have an impact on the standards of acute stroke care and the training of junior staff in this field.

\section{ETHICS STATEMENT}

STREAM is a registered trial at study PI (WP) and all local PIs are https://clinicaltrials.gov/ct2/show/NCT03228251. The trial was approved by the institutional ethics review board of the University Hospital Frankfurt with secondary approval by the local IRBs of all participating centers. The trained according to the needed certification in the context of practice. They are able conduct clinical trials according to Good Clinical Practice (GCP) and the Declaration of Helsinki. The study was funded by Stryker Neurovascular. The funding source was not involved in study design, monitoring, data collection, statistical analyses, interpretation of results, or manuscript writing. The trial results will be disseminated at national and international conferences (Neurowoche, European Stroke Conference) and we aim at publication in an international peerreviewed journal. De-identified patient-level data can be made available upon well-founded request to the corresponding author after clearance with the ethics review board of the University Hospital Frankfurt.

\section{REFERENCES}

1. Emberson J, Lees KR, Lyden P, Blackwell L, Albers G, Bluhmki E, et al. Effect of treatment delay, age, and stroke severity on the effects of intravenous thrombolysis with alteplase for acute ischaemic stroke: a meta-analysis of individual patient data from randomised trials. Lancet. (2014) 384:1929-35. doi: 10.1016/S0140-6736(14)60584-5

2. Saver JL, Goyal M, van der Lugt A, Menon BK, Majoie CB, Dippel DW, et al. Time to treatment with endovascular thrombectomy and outcomes from ischemic stroke: a meta-analysis. JAMA. (2016) 316:1279-88. doi: 10.1001/jama.2016.13647

3. Meretoja A, Keshtkaran M, Saver JL, Tatlisumak T, Parsons MW, Kaste M, et al. Stroke thrombolysis: save a minute, save a day. Stroke. (2014) 45:1053-8. doi: 10.1161/STROKEAHA.113.002910

4. Whiteley WN, Emberson J, Lees KR, Blackwell L, Albers G, Bluhmki E, et al. Risk of intracerebral haemorrhage with alteplase after acute ischaemic stroke: a secondary analysis of an individual patient data meta-analysis. Lancet Neurol. (2016) 15:925-33. doi: 10.1016/S1474-4422(16)30076-X

5. Stotts G, Poppe AY, Roy D, Jovin TG, Lum C, Williams D, et al. Defining the role of the stroke physician during endovascular therapy of acute ischemic stroke. Stroke. (2017) 48:805-7. doi: 10.1161/STROKEAHA.116.015385

6. Strbian D, Ahmed N, Wahlgren N, Lees KR, Toni D, Roffe C, et al. Trends in Door-to-Thrombolysis Time in the Safe Implementation of Stroke thrombolysis registry: effect of center volume and duration of registry membership. Stroke. (2015) 46:1275-80. doi: 10.1161/STROKEAHA.114.007170

7. Kamal N, Sheng S, Xian Y, Matsouaka R, Hill MD, Bhatt DL, et al. Delays in door-to-needle times and their impact on treatment time and

\section{AUTHOR CONTRIBUTIONS}

FB, NK, PRo, HR, and WP: conception of the trial. FB, $\mathrm{NK}$, and WP: drafting of the manuscript and design of the trial intervention. FB, NK, HR, KG, JG, TM, and WP: design of case report form and questionnaire. KG, HR, and FB: central management of process time data. JG, HR, and FB: central management of staff questionnaire data. RM, PZ, ME, $A B$, SP, AM, PRi, SN, JP, FW, LKe, MH, LKo, KH, AA, CS, CB, $\mathrm{CD}, \mathrm{OO}$, and $\mathrm{CK}$ : local data acquisition and analysis at the trial centers. All authors critically revised the manuscript for important intellectual content, provided approval for publication of the content, and agreed to be accountable for all aspects of the work in ensuring that questions related to the accuracy or integrity of any part of the work are appropriately investigated and resolved.

\section{FUNDING}

The study was funded by Stryker Neurovascular. The funding source was not involved in study design, monitoring, data collection, statistical analyses, interpretation of results, or manuscript writing.

\section{SUPPLEMENTARY MATERIAL}

The Supplementary Material for this article can be found online at: https://www.frontiersin.org/articles/10.3389/fneur. 2019.00969/full\#supplementary-material

Supplemental Data 1 | Case report form of the STREAM trial.

outcomes in get with the guidelines-Stroke. Stroke. (2017) 48:946-54. doi: 10.1161/STROKEAHA.116.015712

8. Meretoja A, Strbian D, Mustanoja S, Tatlisumak T, Lindsberg PJ, Kaste M. Reducing in-hospital delay to 20 minutes in stroke thrombolysis. Neurology. (2012) 79:306-13. doi: 10.1212/WNL.0b013e31825d6011

9. Meretoja A, Weir L, Ugalde M, Yassi N, Yan B, Hand P, et al. Helsinki model cut stroke thrombolysis delays to 25 minutes in Melbourne in only 4 months. Neurology. (2013) 81:1071-6. doi: 10.1212/WNL.0b013e3182a $4 \mathrm{a} 4 \mathrm{~d} 2$

10. Kohn LT. To Err is Human: Building a Safer Health System. In: Institute of Medicine (US) Committee on Quality of Health Care in America, Kohn LT, Corrigan JM, Donaldson MS, editors. Washington, DC: National Academies Press (2000).

11. Landrigan CP, Parry GJ, Bones CB, Hackbarth AD, Goldmann DA, Sharek PJ. Temporal trends in rates of patient harm resulting from medical care. $N$ Engl J Med. (2010) 363:2124-34. doi: 10.1056/NEJMsa1004404

12. Makary MA, Daniel M. Medical error-the third leading cause of death in the US. BMJ. (2016) 353:i2139. doi: 10.1136/bmj.i2139

13. Rall M, Schaedle B, Zieger J, Naef W, Weinlich M. Neue trainingsformen und erhöhung der patientensicherheit. Der Unfallchirurg. (2002) 105:1033-42. doi: 10.1007/s00113-002-0523-3

14. Gaba DM. Crisis resource management and teamwork training in anaesthesia. Br J Anaesth. (2010) 105:3-6. doi: 10.1093/bja/aeq124

15. Willems LM, Kurka N, Bohmann F, Rostek P, Pfeilschifter W. Tools for your stroke team: adapting crew-resource management for acute stroke care. Pract Neurol. (2019) 19:36-42. doi: 10.1136/practneurol-2018-001966

16. Tahtali D, Bohmann F, Rostek P, Misselwitz B, Reihs A, Heringer F, et al. (2016). Crew-ressource-management und simulatortraining 
in der akuten schlaganfalltherapie. Der Nervenarzt. 87:1322-31. doi: 10.1007/s00115-016-0162-5

17. Tahtali D, Bohmann F, Kurka N, Rostek P, Todorova-Rudolph A, Buchkremer $\mathrm{M}$, et al. Implementation of stroke teams and simulation training shortened process times in a regional stroke network-A network-wide prospective trial. PLoS ONE. (2017) 12:e0188231. doi: 10.1371/journal.pone.0188231

18. Sexton JB, Helmreich RL, Neilands TB, Rowan K, Vella K, Boyden J, et al. The safety attitudes questionnaire: psychometric properties, benchmarking data, and emerging research. BMC Health Services Res. (2006) 6:44. doi: 10.1186/1472-6963-6-44

19. Zimmermann N, Küng K, Sereika SM, Engberg S, Sexton B, Schwendimann $\mathrm{R}$, et al. Assessing the safety attitudes questionnaire (SAQ), German language version in Swiss university hospitals - a validation study. BMC Health Services Res. (2013) 13:347. doi: 10.1186/1472-6963-13-347

20. Rall M, Gaba D. Human performance and patient safety. In: RD M, editor. Miller's Anesthesia, Philadelphia, PA: Elsevier Churchhill Livingstone (2009). p. 106-66.

21. Neily J, Mills PD, Young-Xu Y, Carney BT, West P, Berger DH, et al. Association between implementation of a medical team training program and surgical mortality. JAMA. (2010) 304:1693-700. doi: 10.1001/jama.2010.1506

22. Ruff IM, Liberman AL, Caprio FZ, Maas MB, Mendelson SJ, Sorond $\mathrm{FA}$, et al. A resident boot camp for reducing door-to-needle times at academic medical centers. Neurol Clin Pract. (2017) 7:237-45. doi: 10.1212/CPJ.0000000000000367

23. Carvalho VS Jr, Picanço MR, Volschan A, Bezerra DC. Impact of simulation training on a telestroke network. Int $J$
Stroke. (2018) 24:1747493018791030. doi: 10.1177/17474930187 91030

24. Mehta T, Strauss S, Beland D, Fortunato G, Staff I, Lee N. Stroke simulation improves acute stroke management: a systems-based practice experience. J Grad Med Educ. (2018) 10:57-62. doi: 10.4300/JGME-D-1700167.1

25. Bohmann FO, Tahtali D, Kurka N, Wagner M, You SJ, du Mesnil de Rochemont $\mathrm{R}$, et al. A network-wide stroke team program reduces time to treatment for endovascular stroke therapy in a regional stroke-network. Cerebrovasc Dis. (2018) 45:141-8. doi: 10.1159/0004 87965

Conflict of Interest Statement: The authors declare that the research was conducted in the absence of any commercial or financial relationships that could be construed as a potential conflict of interest.

Copyright (๑) 2019 Bohmann, Kurka, du Mesnil de Rochemont, Gruber, Guenther, Rostek, Rai, Zickler, Ertl, Berlis, Poli, Mengel, Ringleb, Nagel, Pfaff, Wollenweber, Kellert, Herzberg, Koehler, Haeusler, Alegiani, Schubert, Brekenfeld, Doppler, Onur, Kabbasch, Manser, Pfeilschifter and STREAM Trial Investigators. This is an openaccess article distributed under the terms of the Creative Commons Attribution License (CC BY). The use, distribution or reproduction in other forums is permitted, provided the original author $(s)$ and the copyright owner(s) are credited and that the original publication in this journal is cited, in accordance with accepted academic practice. No use, distribution or reproduction is permitted which does not comply with these terms. 


\section{STREAM COLLABORATORS}

University Hospital Frankfurt, Theodor-Stern-Kai 7, 60590

Frankfurt am Main, Germany

Mohammad Alotaibi, Department of Neurology, University

Hospital Frankfurt

AbdulAziz Batarfi, Department of Neurology, University

Hospital Frankfurt

Dr. Annemarie Brandhofe, Department of Neurology, University

Hospital Frankfurt

Dr. Roxane-Isabelle Kestner, Department of Neurology,

University Hospital Frankfurt

Dr. Jan Hendrik Schaefer, Department of Neurology, University

Hospital Frankfurt

Dr. Martin Alexander Schaller, Department of Neurology,

University Hospital Frankfurt

Dr. Alexander Seiler, Department of Neurology, University Hospital Frankfurt

Stephanie Wallenwein, Department of Neurology, University Hospital Frankfurt

Dr. Laurent M. Willems, Department of Neurology, University

Hospital Frankfurt

Prof. Dr. Helmuth Steinmetz, Department of Neurology, University Hospital Frankfurt

Prof. Dr. Elke Hattingen, Institute for Diagnostic and Interventional Neuroradiology, University Hospital Frankfurt

Klinikum Augsburg, Department of Neurology and Clinical Neurophysiology, Stenglinstr. 286156 Augsburg, Germany

Zeljko Kos, Department of Neurology and Clinical Neurophysiology, Klinikum Augsburg

Prof. Dr. Markus Naumann, Department of Neurology and Clinical Neurophysiology, Klinikum Augsburg

University Hospital Tübingen, Hoppe-Seyler-Str. 3, 72076 Tübingen, Germany

Corinna Blum, Department of Neurology with Focus on Neurovascular Diseases and Neurooncology, and Hertie Institute for Clinical Brain Research, University of Tübingen

Paula Bombach, Department of Neurology with Focus on Neurovascular Diseases and Neurooncology, and Hertie Institute for Clinical Brain Research, University of Tübingen

Julia Zeller, Department of Neurology with Focus on Neurovascular Diseases and Neurooncology, and Hertie Institute for Clinical Brain Research, University of Tübingen

University Hospital Heidelberg, Im Neuenheimer Feld 400, 69120 Heidelberg, Germany

Dr. Christoph Gumbinger, Department of Neurology, University Hospital Heidelberg

Dr. Jens Regula, Department of Neurology, University Hospital Heidelberg

Dr. Solveig Horstmann, Department of Neurology, University Hospital Heidelberg

Dr. Miriam Heyse, Department of Neurology, University Hospital Heidelberg
Dr. Eva Dorozewski, Department of Neurology, University Hospital Heidelberg

Dr. Christian Hamentner, Department of Neurology, University Hospital Heidelberg

Dr. Reiff Tilman, Department of Neurology, University Hospital Heidelberg

Dr. Simon Schieber, Department of Neurology, University Hospital Heidelberg

Dr. Sibu Mundiyanapurath, Department of Neurology, University Hospital Heidelberg

Dr. Silvia Schönenberger, Department of Neurology, University Hospital Heidelberg

Yahia Mokli, Department of Neurology, University Hospital Heidelberg

Dr. Markus Möhlenbruch, Department of Neuroradiology, University Hospital Heidelberg

Ludwig Maximilians-University, Marchioninistrasse 15, 81377 Munich, Germany

Dr. Jan Bewersdorf, Department of Neurology, Ludwig Maximilians-University, Munich

Dr. Maximilian Einhäupl, Department of Neurology, Ludwig Maximilians-University, Munich

Dr. Katharina Feil, Department of Neurology, Ludwig Maximilians-University, Munich

PD Dr. Matthias Klein, Department of Neurology, Ludwig Maximilians-University, Munich

Dr. Ken Möhwald, Department of Neurology, Ludwig Maximilians-University, Munich

Dr. Konstanze Mühlbauer, Department of Anesthesiology, Ludwig Maximilians-University Munich

Dr. Mathias Mulazzani, Department of Neurology, Ludwig Maximilians-University, Munich

Dr. Guido Rohrer, Department of Neurology, Ludwig Maximilians-University, Munich

Dr. Sonja Schönecker, Department of Neurology, Ludwig Maximilians-University, Munich

PD Dr. Franziska Dorn, Department for Diagnostic and Interventional Neuroradiology of the University of Munich (LMU), Campus Grosshadern

Dr. Philipp Mennemeyer, Department for Diagnostic and Interventional Neuroradiology of the University of Munich (LMU), Campus Grosshadern

Dr. Torleif Sandner, Department for Diagnostic and Interventional Neuroradiology of the University of Munich (LMU), Campus Grosshadern

Brigitte Huber, Institute for Stroke and Dementia Research, Ludwig Maximilians-University Munich

Julia Hill, Institute for Stroke and Dementia Research, Ludwig Maximilians-University Munich

Jela Gavran, Emergency Department, Klinikum Grosshadern, Ludwig Maximilians University, Munich

Charité - University Hospital Berlin, Center for Stroke Research Berlin (CSB), Hindenburgdamm 30, 12203 Berlin

Prof. Dr. Heinrich Audebert, Department of Neurology, Charité - University Hospital Berlin 
Rohat Geran, Center for Stroke Research Berlin (CSB), Charité - University Hospital Berlin

Johannes Schurig, Center for Stroke Research Berlin (CSB), Charité - University Hospital Berlin

Dr. Juliane Herm, Center for Stroke Research Berlin (CSB), Charité - University Hospital Berlin

Felix Kleefeld, Center for Stroke Research Berlin (CSB), Charité

- University Hospital Berlin

Dr. Karl Schoknecht, Center for Stroke Research Berlin (CSB), Charité - University Hospital Berlin

Denes Jadranka, Center for Stroke Research Berlin (CSB), Charité - University Hospital Berlin

Kirsten Brade, Center for Stroke Research Berlin (CSB), Charité - University Hospital Berlin

Tatjana Wittenberg, Center for Stroke Research Berlin (CSB), Charité - University Hospital Berlin

University Medical Centre Hamburg Eppendorf, Martinistr. 52, 20246 Hamburg, Germany

Ulrich Mayer-Runge, Central Emergency

Department, University Medical Centre Hamburg Eppendorf, Hamburg

Maxim Bester, Department of Diagnostic and Interventional Neuroradiology, University Medical Centre Hamburg Eppendorf, Hamburg

Michael H Schönfeld, Department of Diagnostic and Interventional Neuroradiology, University Medical Centre Hamburg Eppendorf, Hamburg

Fabian Flottmann, Department of Diagnostic and Interventional Neuroradiology, University Medical Centre Hamburg Eppendorf, Hamburg

Lisa Prilop, Department of Neurology, University Medical Centre Hamburg Eppendorf, Hamburg

Hannes Leischner MD PhD, Department of Diagnostic and Interventional Neuroradiology, University Medical Centre Hamburg Eppendorf, Hamburg

Andreas Maximilian Frölich, Department of Diagnostic and Interventional Neuroradiology, University Medical Centre Hamburg Eppendorf, Hamburg

Sabine Roesner, Department of Neurology, University Medical Centre Hamburg Eppendorf, Hamburg

Gabriel Broocks, Department of Diagnostic and Interventional Neuroradiology, University Medical Centre Hamburg Eppendorf, Hamburg

Uta Hanning, MD, Department of Diagnostic and Interventional Neuroradiology, University Medical Centre Hamburg Eppendorf, Hamburg

Stephanie Guder, Department of Neurology, University Medical Center Hamburg Eppendorf, Hamburg

Dr. med. Matthias Bechstein, Department of Diagnostic and Interventional Neuroradiology,
University Medical Centre Hamburg

Eppendorf, Hamburg

Caspar Brekenfeld, Department of Diagnostic and Interventional Neuroradiology, University Medical Centre Hamburg Eppendorf, Hamburg

Carmen Lange, Department of Neurology, University Medical Center Hamburg Eppendorf, Hamburg

Sebastian Kautz, Department of Diagnostic and Interventional Neuroradiology, University Medical Centre Hamburg Eppendorf, Hamburg

Focko L. Higgen, Department of Neurology, University Medical Center Hamburg-Eppendorf, Hamburg, Germany

Dr. Anna Kyselyova, Department for Neuroradiological Diagnosis and Intervention, University Medical Center Hamburg-Eppendorf

Prof. Dr. Götz Thomalla, Department of Neurology, University Medical Center Hamburg-Eppendorf, Hamburg, Germany

Prof. Dr. Jens Fiehler, Department of Diagnostic and Interventional Neuroradiology, University Medical Centre Hamburg Eppendorf, Hamburg

\section{University Hospital Cologne, Kerpener Str. 62, 50937 Cologne, Germany}

Prof. Dr. Gereon Rudolf Fink, Department of Neurology, University Hospital Cologne

Anna Bonkhoff, Department of Neurology, University Hospital Cologne

Dr. Julian Dronse, Department of Neurology, University Hospital Cologne

Dr. Katharina Kirsch, Department of Neurology, University Hospital Cologne

Sarah Laurent, PhD, Department of Neurology, University Hospital Cologne

Dr. Boris von Reutern, Department of Neurology, University Hospital Cologne

Dr. Jurij Rosen, Department of Neurology, University Hospital Cologne

Dr. Lukas Volz, Department of Neurology, University Hospital Cologne

Jan-Michael Werner, Department of Neurology, University Hospital Cologne

Michael Wollring, Department of Neurology, University Hospital Cologne

Robert Seliger, Department of Neurology, University Hospital Cologne

Abdulkadir Yildirim, Department of Neurology, University Hospital Cologne

Prof. Dr. Marc Schlamann, Department of Neuroradiology, University Hospital Cologne

PD Dr. Jan Borggrefe, Department of Neuroradiology, University Hospital Cologne 